\title{
A Proposed Method to Measure Sub Pixel Shift in Egyptsat-1 Aliased Images
}

\author{
S.A. Mohamed \\ Data Reception, Analysis \\ and Receiving Station \\ Affairs \\ National Authority for \\ Remote Sensing and \\ Space Sciences \\ Cairo, Egypt
}

\author{
A.K. Helmy \\ Data Reception Analysis \\ and Receiving Station \\ Affairs \\ National Authority for \\ Remote Sensing and \\ Space Sciences \\ Cairo, Egypt
}

\author{
M.A. Fkirin \\ Department of Industrial \\ Electronic Engineering and \\ Control \\ Faculty of Electronic \\ Engineering, \\ Menoufia University \\ Cairo, Egypt
}

\author{
S.M. Badway \\ Department of Industrial \\ Electronic Engineering and \\ Control \\ Faculty of Electronic \\ Engineering, \\ Menoufia University \\ Cairo, Egypt
}

\begin{abstract}
This paper presents a method for image-based shift measurement and investigates solution for the mismatched bands of egyptsat-1 satellite. A tiny error in measuring sub pixel shift leads to an incorrect image focusing. Different methods, using frequency approach are conducted with different Tukey window sizes for measuring the sub pixel shift. The present method use an aim to select most important frequency for three parameter; horizontal, vertical and rotation with different window size. To test the present method; we used the SPOT-5 satellite data with known displacement. The obtained results are acceptable, and might solve Egyptsat-1 problem of mismatches between its bands. Bands 1, 2 could also be used in super resolution reconstruction methods
\end{abstract}

\section{General Terms}

Image processing, Remote sensing

\section{Keywords}

Spot-5; sub-pixel shift; Tukey windows; window size; superresolution

\section{INTRODUCTION}

The Egyptian satellite (Egyptsat-1) is the first earth observation satellite to Egypt. It was launched in April 2007, based on micro-satellite technology. The satellite is intended to image certain areas of ground and transmit its images to the ground data receiving station. That satellite is consists of four payload subsystems; Multiband Earth Imager (MBEI), Middle IR Earth Imager (MIREI), Store and forward communication and command and data handling. Beside the extend observation capabilities (+/-35 degree) off-nadir cross-track satellite pointing. It provided imaging in each of the modes Panchromatic (PAN), Multispectral (MS) and Infrared (IR). The characteristic of Egyptsat-1 data are as follows, according to A. Kolokolov et al [1]; the spatial resolutions of the MS and the PAN bands are 7.8 meters. The spatial resolution of the Mid-Infrared band is 39.5 meter [2], [3], and the spectral resolutions are as shown in following Table (1).

Table .1 The Spectral resolutions of the Egyptsat-1 Data

\begin{tabular}{|c|c|c|}
\hline Bands & Description & Wavelength $(\boldsymbol{\mu m})$ \\
\hline Band1 & Green & $0.51-0.59$ \\
\hline Band2 & Red & $0.61-0.68$ \\
\hline Band3 & Near Infrared & $0.80-0.89$ \\
\hline Band4 & Panchromatic & $0.50-0.89$ \\
\hline Band5 & Mid Infrared & $1.10-1.70$ \\
\hline
\end{tabular}

Receiving station has received images of Egyptsat-1 since 2007. While images were being analyzed, found bands unregister between them. Bands unregister have showed since 2010, show in fiuger.1. After had analyzed images, bands mismatched [4].Comprehensive analysis was being assessed some method by previous literature $[4,5]$. In previous analyzes were analyzed only the vertical and horizontal. But in this method you will be using a new factor of rotation. In order to do the precise analysis, must be analyzed three parameters; horizontal, vertical shifts $(\Delta \mathrm{x}$ and $\Delta \mathrm{y})$, and a planar rotation angle $\varphi[6,7,8]$.

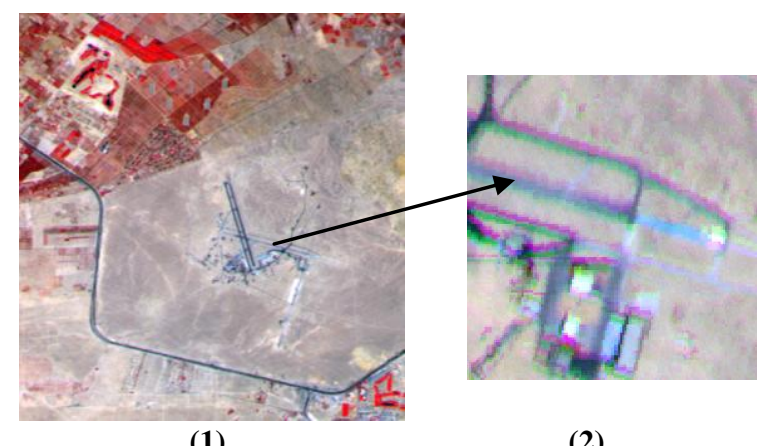

(1)

(2)

Fig 1: (1) Egyptsat-1 Bands (Green, Red, Near Infrared);

(2) Clarify the mismatch between Bands (green and magenta between edges shows problem)

duo to shift between bands, aliasing have been explained after combination bands . Figure.1.2 show this result. The next section will be explained aliasing phenomena.

\section{- $\quad$ Aliasing and Circular Extension}

The described problem shift in egyptsat- 1 bands estimation methods work perfect on noise-free images. Practically images are degraded by noise and aliasing, which often causes failure of the image registration Assume the images, with maximal frequency fmax, are sampled at frequency fs.

$$
\text { With } \mathrm{f}_{\max }<\mathrm{fs}<2 \mathrm{f}_{\max } \text { and all } \mathrm{f}=\left[\begin{array}{l}
\mathrm{fx} \\
\mathrm{fy}
\end{array}\right] \text {. }
$$

This setup does not satisfy the Nyquist criterion (fs > 2fmax) greater than or equal, and therefore aliasing artifacts been present in the sampled images. But, as fs $>$ fmax, the signal is free of aliasing and thus the same in all images (up to shift and rotation effects) at frequencies

$$
-\mathrm{fs}+\mathrm{fmax}<\mathrm{f}<\mathrm{fs}-\text { fmax. }
$$


therefore, the image shift estimation based on these frequencies and use the complete frequency content to reconstruct the high resolution image accurately. This principle has already been applied to image registration [9, 10].Another cause of artifacts in the Fourier transform of images is the fact that they are (in general) not circular. When computing the FFT of an image, it is circularly extended, which causes strong artificial edges in the spatial domain image. In the frequency domain [10], this mainly causes artifacts around the two axes. In order to eliminate this effect, we multiply the images by a Tukey window prior to shift estimation [11]. Tukey window functions yield lower root mean square error (RMSE) [12]. The border lines of the image all have zero value and the image is successfully circularized. This paper uses proposed technique; variable Tukey window from two pixels to 300 pixels. Then, calculate the Sub-Pixel shift between pixels it is required to calculate the rotation between them to get the correct robust result [13]. A variable number of pixels (2:300 pixels) have been used to eliminate the edge effects $[11,14]$. This change in the number of pixels used clarify what is appropriate for use in the calculation process. Figure. 2 shows the calculation steps of Sub-Pixel shift with calculated rotations.

\section{IMAGE SHIFT ESTIMATION}

Frequency domain approach allows us to estimate the horizontal and vertical shift and the planar rotation separately from the most important frequencies of images. Given a reference image $f_{1}(x, y)$. Its shifted and rotated version $f_{2}(x$, y) would be represented as follows:

$$
\mathrm{f}_{2}(\mathrm{X})=\mathrm{f}_{1}(\mathrm{R}(\mathrm{X}+\Delta \mathrm{X})),
$$

$$
\text { With } X=\left[\begin{array}{l}
x \\
y
\end{array}\right], \quad \Delta \mathrm{X}=\left[\begin{array}{l}
\Delta x \\
\Delta y
\end{array}\right], \mathrm{R}=\left[\begin{array}{rr}
\cos \emptyset & -\sin \emptyset \\
\sin \emptyset & \cos \emptyset
\end{array}\right]
$$

Where $\Delta \mathrm{x}$ the Horizontal shift $\Delta \mathrm{y}$ is the Vertical shift and $\mathrm{R}$ is the rotation angle. In this case, the images will be measured for displacement and rotation angle. Bernd Jähne, and Steven T. Karris application of the Fourier Transform properties on our measured images to calculate displacement and rotation angles $[15,16]$ as follows:

$$
\begin{aligned}
\mathrm{F}_{2}(\mathrm{u}) & =\iint_{X} f_{2}(X) e^{-j 2 \pi u^{T} X} d X \\
& =\iint_{X} f_{1}\left(R(X+\Delta X) e^{-j 2 \pi u^{T} X} d X\right. \\
& =e^{j 2 \pi u^{T} \Delta X} \iint_{X} f_{1}(R \dot{x}) e^{-j 2 \pi u^{T} X^{\prime}} d \dot{X}
\end{aligned}
$$

Which depend on the shifts and is a rotated version of $\left|F_{1}(u)\right|$. It is therefore possible to estimate the relative rotation angle between two images first (i.e., Egyptsat-1 mismatch bands). then apply the inverse rotation to the Fourier transform. The (phase) shift can be computed from the resulting image and the reference image.

\subsection{Shift Estimation (Horizontal \& Vertical)}

A shift in the image plane corresponds to a phase shift in the frequency domain. For an image $f_{1}(x)$ and its shifted version $\mathrm{f}_{2}(\mathrm{x})$, with Fourier transforms $\mathrm{F}_{1}(\mathrm{u})$ and $\mathrm{F}_{2}(\mathrm{u})$, respectively, are given by:

$$
\begin{gathered}
F_{2}(u)=e^{j 2 \pi u^{T} \Delta x} \iint_{x} f_{1}(x) e^{-j 2 \pi u^{T} x} d x \\
=e^{j 2 \pi u^{T} \Delta x} F_{1}(u)
\end{gathered}
$$

Thus, the difference between the phases of the two Fourier transforms is a plane in $\mathrm{u}-\mathrm{v}$ space. Horizontal and vertical shifts can therefore be directly computed as the slope of this plane in horizontal and vertical direction, respectively. This phase difference is only approximately a plane. therefore one can solve this problem by estimating the plane parameters from the data with a least squares method to make the estimates more robust to errors.

\subsection{Rotation Estimation}

An image was rotated an angle which produced rotated image .to estimate the rotation angle between the two images (Egyptsat-1 mismatch bands), the computed correlation between the reference image and rotated versions of the second image estimates the rotation angle between the two images. The estimated rotation angle is the angle for which a maximum correlation is obtained [17]. The main disadvantages of this method are its computational cost and the interpolation errors that result from every rotation [18]. The rotation angle between $\left|\mathrm{F}_{1}(\mathrm{u})\right|$ and $\left|\mathrm{F}_{2}(\mathrm{u})\right|$ will be computed as the angle $\theta$ for which the Fourier transform of the reference image $\left|\mathrm{F}_{1}(\mathrm{u})\right|$ and the rotated Fourier transform of the image to be registered $\left|F_{2}(R \theta u)\right|$ have maximum correlation. This implies the computation of a rotation of $\left|F_{2}(u)\right|$ for every evaluation of the correlation, which is computationally heavy and thus practically difficult. If $\left|\mathrm{F}_{1}(\mathrm{u})\right|$ and $\left|\mathrm{F}_{2}(\mathrm{u})\right|$ are transformed into polar coordinates, the rotation over the angle $\theta$ is reduced to a (circular) shift over $\theta$. It can compute the Fourier transform of the spectra $\left|\mathrm{F}_{1}(\mathrm{u})\right|$ and $\mid \mathrm{F}_{2}$ $(\mathrm{u}) \mid$, and compute $\theta$ as the phase shift between the two [19, 20].

\section{THE PROPOSED METHOD}

In this method, Fourier transform is used to calculate weighty range of frequencies based on the hypothesis that the analyzed signal is noise free and the selected frequencies followed the following equation:

$$
\left\|\frac{\sqrt{N}(F(w)-M e a n F(w))}{\operatorname{Std} F(w)}\right\|>f_{N-1,0.005}
$$

Where $\mathrm{N}$ is the number of pixels, $\mathrm{F}(\mathrm{w})$ is the Fourier transform image, Std is the standard deviation of Fourier transform, Mean is the average value of a signals and $\mathrm{fN}-1$ is the most important frequencies.

The intuition of this method is that if we suppose $\mathrm{F}(\mathrm{w})$ are normally distributed, we pick the frequencies that contribute to the Fourier transform "Significantly" at greater than 0.005 level (we miss an important frequency with a probability less than 0.005) . After pick up the significant frequencies [21], finally use amplitude of Fourier transforms to approximate the true signal in Tukey windows. The following simulations will also give clear demonstration of our procedures using Spot-5 and Egyptsat-1 satellite data. The next steps will explain how the proposed method operations:

- Peak value: Get the peak value from the two images for $\mathrm{N}$ size of Tukey windows.

- Mean value: Calculate Mean value from $\left(\mathrm{x}_{0}, \mathrm{y}_{\mathrm{o}}\right)$ to $\left(\mathrm{x}_{\mathrm{n}-1}, \mathrm{y}_{\mathrm{n}-1}\right), \mu \mathrm{x}, \mathrm{y}=\frac{1}{\mathrm{~N}} \sum_{\mathrm{i}, \mathrm{j}=0}^{\mathrm{N}-1} \mathrm{xi}, \mathrm{yj}$ then, calculate RMS value for the mean values $\mathrm{x}, \mathrm{y}$.

- Standard deviation $(\delta)$ : Calculate $\delta$ for $\mathrm{N}$ samples in Tukey window (xi, yj). $\delta \mathrm{x}^{2}=\frac{1}{\mathrm{~N}-1} \sum_{\mathrm{i}=0}^{\mathrm{N}-1}(\mathrm{xi}-\mu \mathrm{x})$, 
$\delta \mathrm{y}^{2}=\frac{1}{\mathrm{~N}-1} \sum_{\mathrm{i}, \mathrm{J}=0}^{\mathrm{N}-1}(\mathrm{yj}-\mu \mathrm{y})$ and calculate RMS value for $\delta$ value of $\mathrm{x}, \mathrm{y}$.

- Calculate Rotation (R): compute the Fourier transform of the spectra two images, and compute $\mathrm{R}$ as the phase shift between the two selecting frequencies in Tukey window.

Calculate Shift (Horizontal \& Vertical): Multiply $\left(\mathrm{R}^{-1}\right)$ in image_1 $\mathrm{R}^{-1} \mathrm{f}_{1}(\mathrm{x}, \mathrm{y})=\mathrm{f}_{2}\left(\left(\mathrm{x}+\Delta_{\mathrm{X}}\right),\left(\mathrm{y}+\Delta_{\mathrm{y}}\right)\right)$ then get $\Delta_{\mathrm{X}}, \Delta_{\mathrm{y}}$ values.

\section{MATERIALS}

\subsection{Data sets}

Two types of data are used Spot-5 and Egyptsat-1. In Spot-5 the displacement values in the horizontal and vertical direction are defined in the SPOT-5 mission $[4,20]$. The second type Egyptsat-1 in which the displacements are to be measured

\subsubsection{Spot-5}

The French satellite Spot-5 which was launched in 2002. It has a resolution of $5 \mathrm{~m}$ for the panchromatic band (HRG instrument) with an unchanged swath of $60 \mathrm{~km} \mathrm{[14].}$ Extracting the $2.5 \mathrm{~m}$ from $5 \mathrm{~m}$ images requires a special technique. We have used tow scenes definitely shifted $(0.5$, 0.5 ) with characteristics are shown in Table 2 , as simulation method.

Table.2 Spectral characteristics of the Spot-5 Data.

\begin{tabular}{|c|c|c|}
\hline Images & Image_1 & Image_2 \\
\hline $\mathrm{K}$ & 113 & 113 \\
\hline $\mathrm{J}$ & 289 & 289 \\
\hline Description & Panchromatic & Panchromatic \\
\hline Wavelength $(\mu \mathrm{m})$ & $0.48-0.71$ & $0.48-0.71$ \\
\hline Spectral mode & $\mathrm{A}$ & $\mathrm{B}$ \\
\hline Processing level & $1 \mathrm{~A}$ & $1 \mathrm{~A}$ \\
\hline Acquisition date & $16 / 06 / 2008$ & $16 / 06 / 2008$ \\
\hline
\end{tabular}

\subsubsection{Egyptsat-1}

The image acquisition was on March 2010 and it consists of three bands (Green, Red and near infrared). Their characteristics are shown in Table 3

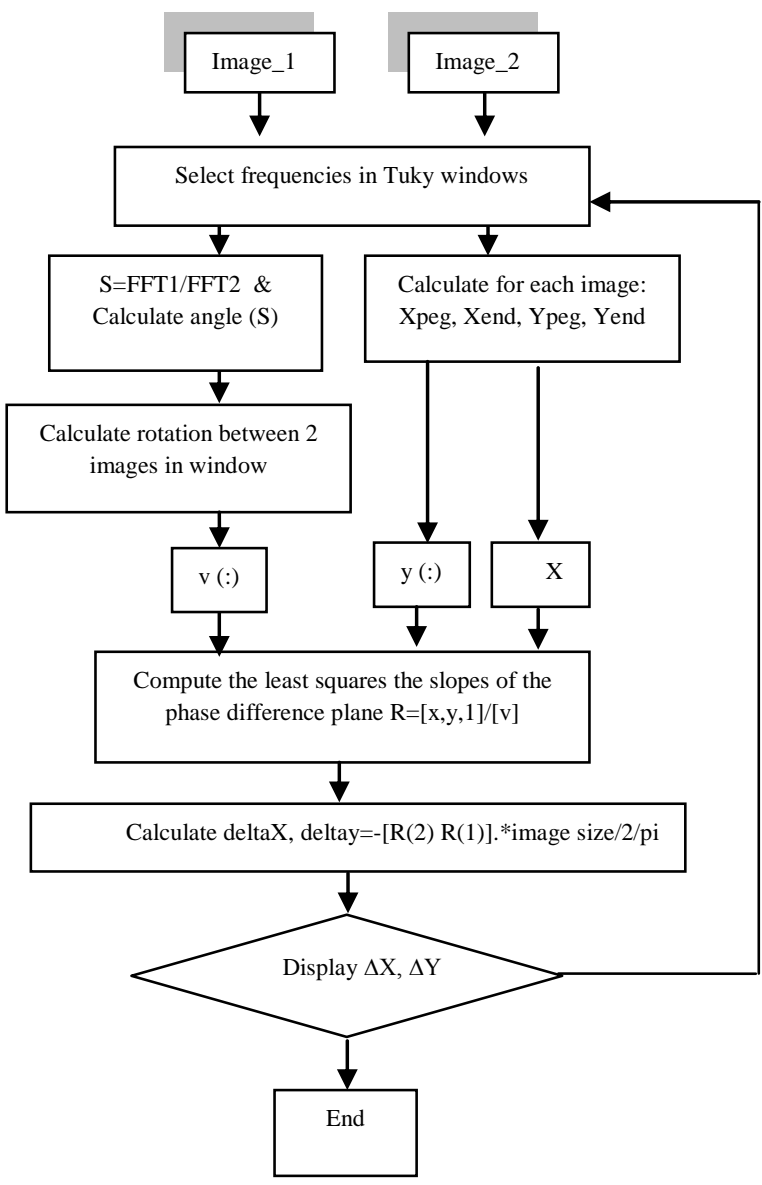

Fig 2 Workflow diagram of the proposed Sub-Pixel shift based different Tukey window size method

Table.3 Spectral characteristics of Egyptsat-1

\begin{tabular}{|c|c|c|c|}
\hline Bands & Band1 & Band2 & Band3 \\
\hline Description & Green & Red & $\begin{array}{c}\text { Near } \\
\text { Infrared }\end{array}$ \\
\hline $\begin{array}{c}\text { Wavelength } \\
(\mu \mathrm{m})\end{array}$ & $0.51-0.59$ & $0.61-0.68$ & $0.80-0.89$ \\
\hline $\begin{array}{c}\text { Processing } \\
\text { level }\end{array}$ & $1 \mathrm{~A}$ & $1 \mathrm{~A}$ & $1 \mathrm{~A}$ \\
\hline $\begin{array}{c}\text { Acquisition } \\
\text { date }\end{array}$ & \multicolumn{3}{|c|}{$31 / 05 / 2010$} \\
\hline
\end{tabular}

\section{RESULTS}

The previously reported method was tested in a simulation setup as well as in a practical experiment. The goal of the simulation was to evaluate the performance in a controlled environment with exact knowledge about the shift and rotation values from selected most important frequencies. This method is very interacting in biomedical imaging, remotesensing, surface inspection and face recognition [21]. In the first step used satellite images with known sub-pixel shift Spot-5. And in the second step we used Egyptsat-1 satellite images to calculate the sub-pixel shift. 


\subsection{Spot-5 Results}

The previous algorithm mentioned in section 3 in order to simulate the sub pixel shift using a couple of panchromatic images $(2000 \times 2000$ pixels $)$ of spot-5, by using different window sizes on shifting image. Figure 3 shows the two images which have "-0.5, 0.5 pixels" shift between them. There are many different pixels angle in the images that will affect in analysis horizontal and vertical shift in the results as show in Figure 4-a. But in this part of the analysis we needed to use the difference in the rotation between the two images to empower the testing of robust. The robust test will yield construct an accurate shift values that can be used in super resolution reconstruction process $[22,23]$. The output from the analysis of spot-5 satellite images explains Figure 3 that; for satellite images with known Sub-Pixel shift, when we use the values of rotation it gives results closer to reality. And with the change of the window size, it is became clear that the several 100 pixels output of Sub-Pixel shift horizontal and vertical fixed at value $(-0.5,0.5$ pixels). Figure 4-b shows the difference in the rotation between the two images of the spot5 .

\subsection{Egyptsat-1 Result}

Egyptsat-1 image shown in Figure 5, $(2000 \times 2000$ pixels $)$ clearly shows the bands mismatch problem very strongly. This problem gives inaccurate image results in the field (image processing) of remote sensing. The analysis process gives results that are close to reality as shown in the output (figures 6,7, and 8). The three bands of Egyptsat-1 were analyzed and the results were as follows:

\subsubsection{Bands 1, 2}

The results of analyzing Bands 1, 2 and are shown in Figure 6 (a), (b) by following the steps outlined in Figure 2. Results show that the difference in rotation between the two bands are $(-2.5,1.7)$ and also shows the importance of rotational account to access for robust analysis and it is illustrated in Figure 6-b. In addition to, changes as a result of using the Tukey window to get to the most suitable value becomes true solution because it reduces the effect of speckles that produced beside edges. Figure 6-a shows that the most appropriate value that has been reached is (100), which then stabilized. Shift bands described in horizontal and vertical are stabilized at (0.3, 0.6), this is shown in Figure 6-a.

\subsubsection{Bands 2, 3}

The results of Bands 2, 3 showed that the rotation in this part is limited between $(-3,3)$ and it is illustrated in Figure 7-b. But increasing the rotation values led to instability and brought stability after 250 pixels, as shown in Figure 7-a. The result after stability were " $0.6,-0.3 "$.

\subsubsection{Bands 1, 3}

The results here showed that the rotation had a high value (2.4), but then receded between $(0.9,-1)$ as shown in Figure 8b. Decline of values led to the stability of the rotation too fast to measure the difference and this happened before 50 pixels, as shown in Figure 8-a. Also, the figure shows that there is no difference between Band 1 and Band 3 .
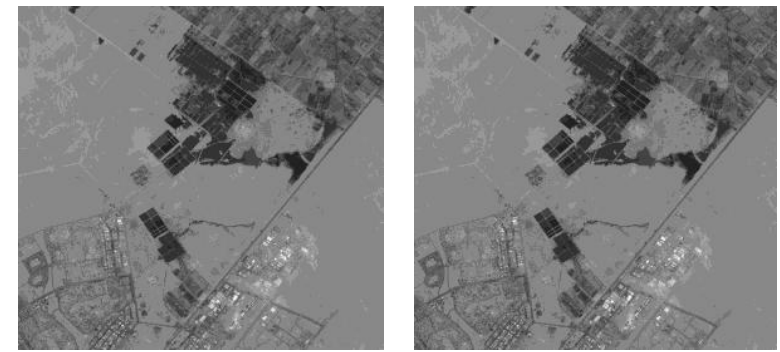

Fig 3. Two images with sup-pixels shift of "-0.5, 0.5 " pixel

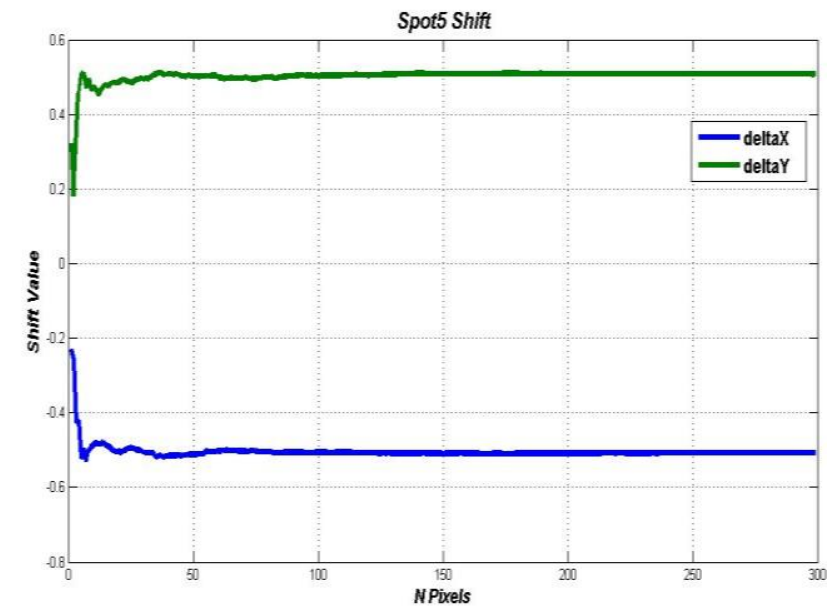

(a)

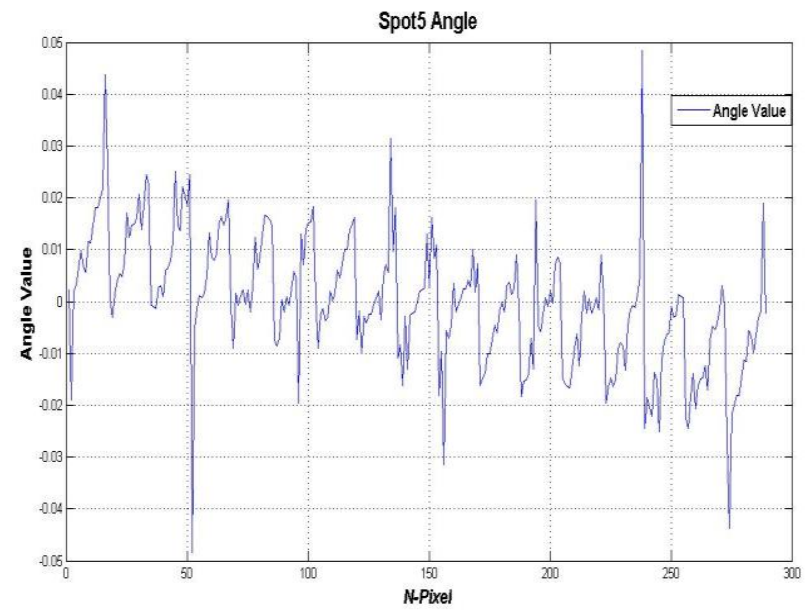

(b)

Fig 4: Shows: "(a) Result of spot-5 Sub-Pixel shift (delta.x, delta.y), (b) Result of rotation variation between images" 

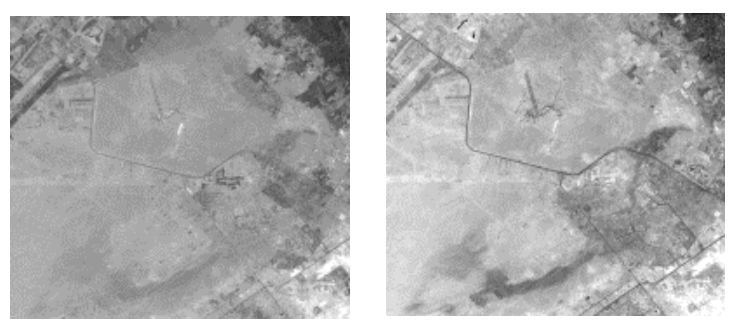

(a)

(b)

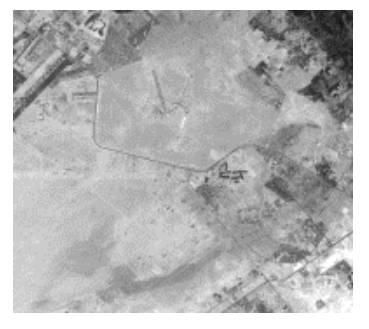

Fig 5: Egyptsat-1 bands: (a) Band-1 Green (b) Band-2 Red (c) Band-3 Near Infrared

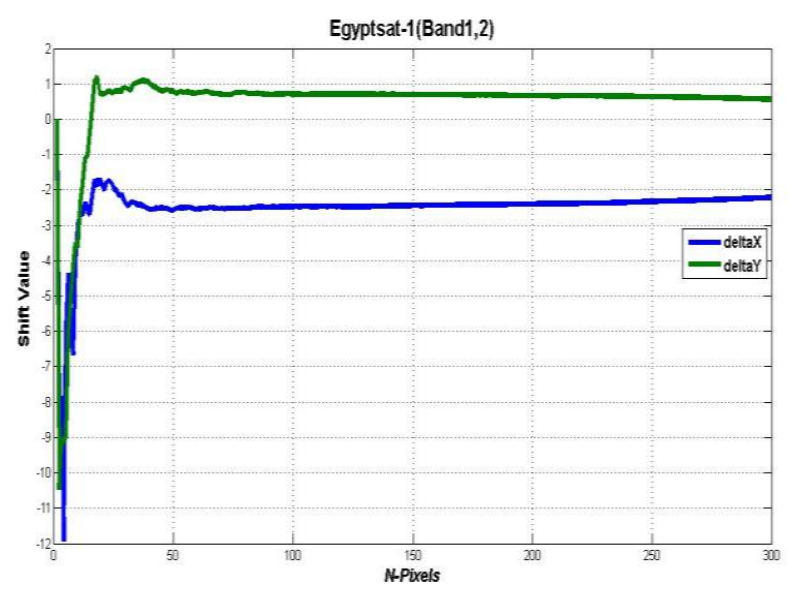

(a)

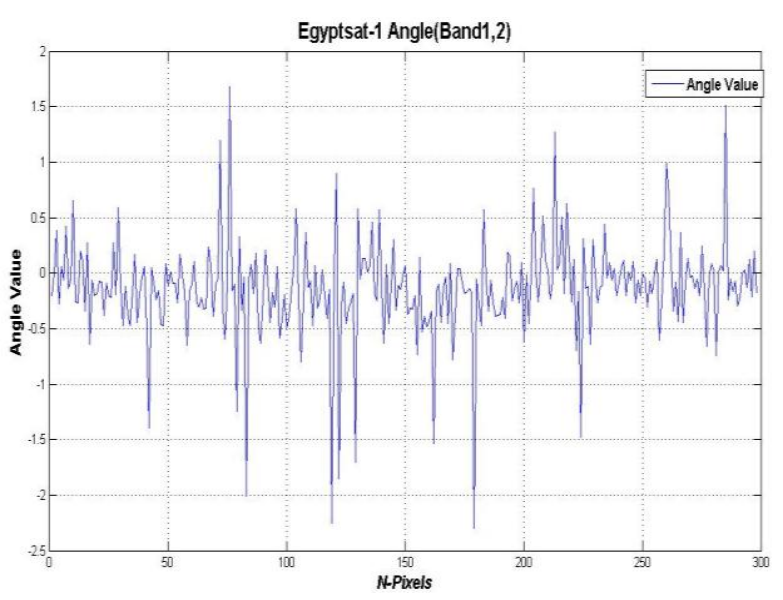

(b)

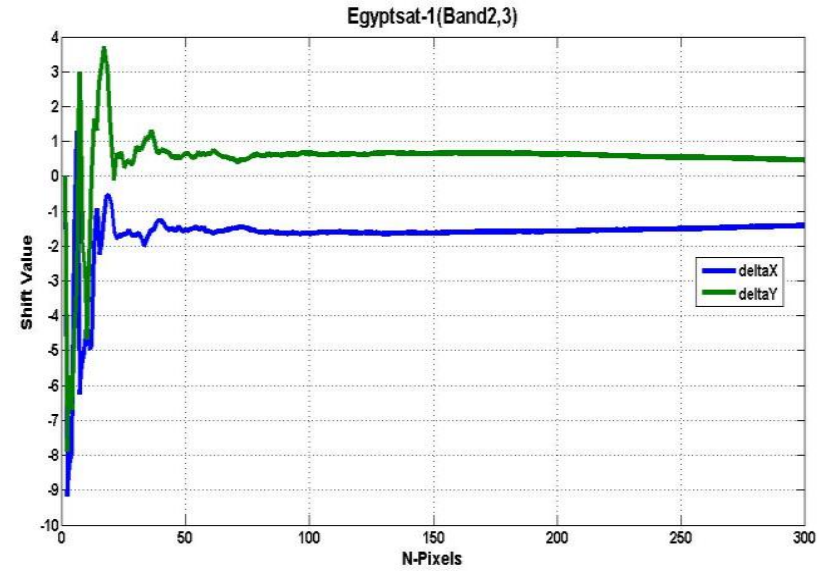

(a)

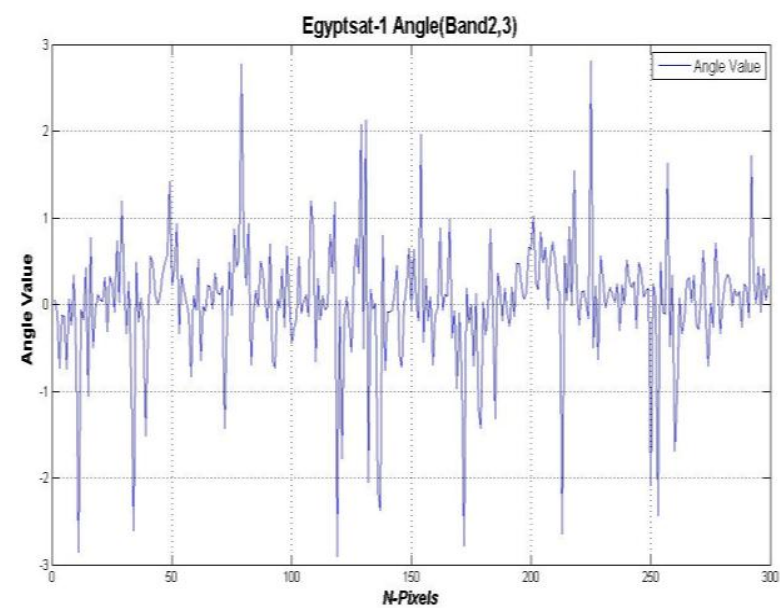

(b)

Fig 7: Shows "(a) Result of Egyptsat-1bands 2, 3 Sub-Pixel shift (delta.x, delta.y), (b) Result of rotation variation between images".

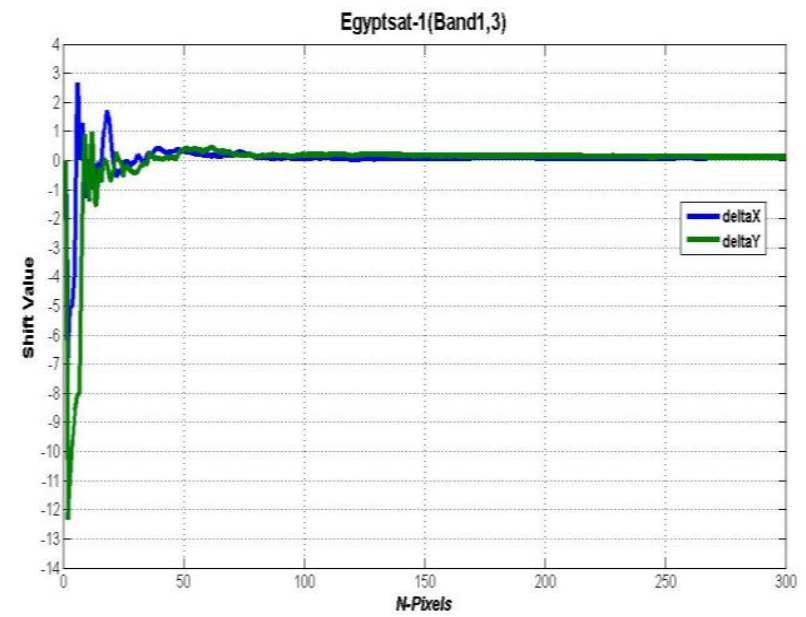

(a)

Fig 6: shows "(a) Result of Egyptsat-1bands 1, 2 Sub-Pixel shift (delta.x, delta.y), (b) Result of rotation variation between images" 


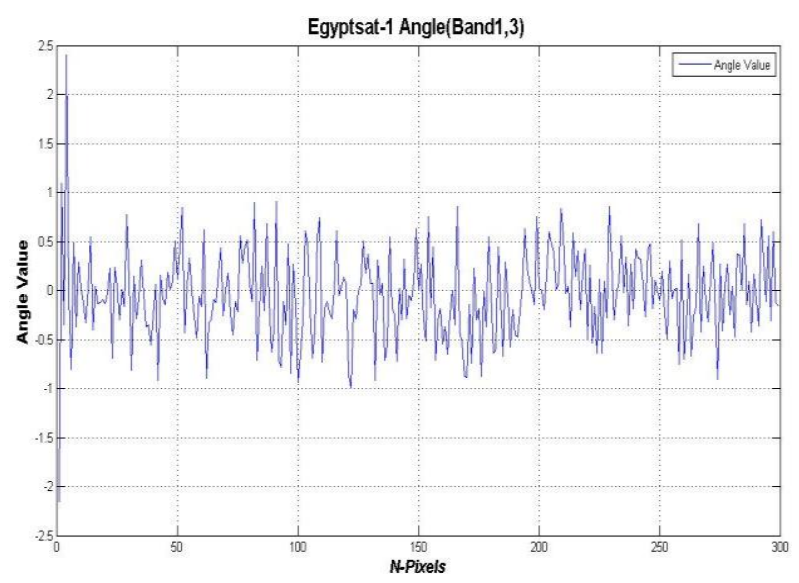

(b)

Fig 8:Shows "(a) Result of Egyptsat-1 bands 1, 3 Sub-Pixel shift (delta.x, delta.y), (b) Result of rotation variation between images"

\section{CONCLUSION}

This paper found a proper solution to the mismatches between the Egyptsat-1 satellite Bands. The proposed method is to select the most important frequencies for all bands using different sizes of Tukey window and to calculate the optimum rotation and Sub-Pixel shift at every window size. the results show more accuracy in estimation of small planar motion parameters, using the proposed algorithm .also results show Increasing the value of the rotation affect the final solution to calculate the Sub-Pixel shift, and also describes the problem of mismatches occurred from band-2, As band 1,3 are identical. Consequence, this method could be used in image registration to reconstructing a super resolution images for Egyptsat-1.

\section{ACKNOWLEDGMENT}

The authors are grateful to the National Authority for Remote Sensing and Space Sciences (NARSS) for supporting this study. Special thanks are extended to the anonymous reviewers, whose comments were particularly valuable and their efforts are greatly appreciated.

\section{REFERENCES}

[1] A. Kolokolov.,G. Tarasov.,S. Medvednikone.,V. Gladilin.,"Egyptsat-1 satellite specification" NARSS technology transfer documents., Issue 3, pp13-26, 2006.

[2] Nasr, A.H., Helmy, A.K. Mohamed, S.A., "Exploration of Misrsat-1 Data in Different Change Detection Applications". Proc. of the 5th WSEAS International Conference on Remote Sensing (REMOTE'09), University of Genova, Genova, Italy, pp.39-46, 2009.

[3] Nasr, A.H. and Helmy, A.K., "Egyptsat-1 SuperResolution Image Reconstruction Using Data Fusion". Proc. of the International Joint Urban Remote Sensing Event (JURSE-2011), Technische Universitat Munchen, Munich, Germany, PP. 317-320, 2011.

[4] S.A. Mohamed; A.K. Helmi ; M.A. Fkirin; S.M. Badwai, "Subpixel Accuracy Analysis of Phase Correlation Shift Measurement Methods Applied to Satellite Imagery"(IJACSA) International Journal of Advanced Computer Science and Applications, ISSN21565570, Vol. 3, No. 12, pp. 202-206, 2012.
[5] S.A. Mohamed; A.K. Helmi ; M.A. Fkirin; S.M. Badwai" Accuracy Analysis of Phase Correlation Shift Measurement Methods Applied to Egyptsat-1 Satellite" Radio Science Conference (NRSC), 2013 30th National ,ISBN 978-1-4673-6219-1,PP: 347-358,Cairo,Egypt ,1618 April2013

[6] P. Vandewalle, S. S $\square$ usstrunk, and M. Vetterli, "Superresolution images reconstructed from aliased images," in SPIE / IS \& T Visual Communication and Image Processing Conference, T. Ebrahimi and T. Sikora, eds., 5150, pp. 1398\{1405, July 2003.

[7] Jian Li, Student Member, IEEE, and Rama Chellappa, Fellow, IEEE " Structure From Planar Motion" IEEE Transactions on Image Processing, Vol. 15, No. 11, November, 2006.

[8] L. Lu, H.T. Tsui and Z. Hu, "A Novel Planar Motion Detection Method and the Robust Estimation of 1D Trifocal Tensor", ICPR 2000, Vol. 3, pp. 815-818, Sep. 2000, Barcelona, Spain.

[9] S. P. Kim and W.-Y. Su, "Subpixel accuracy image registration by spectrum cancellation," in Proceedings IEEE International Conference on Acoustics, Speech and Signal Processing, 5, pp. 153\{156, April 1993.

[10] Frederic Truchetet and Olivier Laligant, "Review of industrial applications of wavelet and multiresolution based signal and image processing", Journal of Electronic imaging, SPIE and IS \& T, 2008.

[11] L. M. Surhon, M. T. Timpledon, S. F. Marseken, "window Function" ISBN 613030014X, 9786130300142, VDM Verlag, p.124, 2010.

[12] Arora, D.; Felix, D.; McGuire, M. "Reducing the error in mobile location estimation using robust window functions "IEEE Conference Publications. ISBN978-14244-4560-8 , Victoria, BC , pp. 199-204, 23-26 Aug. 2009.

[13] Pohl, Nils ; Jaeschke, Timo ; Vogt, Michael "Ultra high resolution SAR imaging using an $80 \mathrm{GHz}$ FMCW-radar with $25 \mathrm{GHz}$ bandwidth" Synthetic Aperture Radar, 2012. EUSAR. 9th European Conference on, pp. 189$192,2012$.

[14] C. Fratter, M. Moulin, H. Ruiz, P. Charvet, D. Zobler, "The SPOT-5 mission", 52nd International Astronautical Congress, Toulouse, France, 1-5 Oct 2001.

[15] Bernd Jähne, "Digital image processing" 6th Edition, ISBN 3-540-24035-7 Springer Berlin, Heidelberg, New York, 2005

[16] Steven T. Karris, "Signals and Systems with MATLAB Applications, Second Edition", Orchard Publications, ISBN 0-9709511-8-3, 2003.

[17] B. S. Reddy and B. N. Chatterji, "An FFT-based technique for translation, rotation, and scale-invariant image registration," IEEE Transactions on Image Processing, vol. 5, no. 8, pp. 1266-1271, 1996.

[18] Kourosh Jafari-Khouzani and Hamid Soltanian-Zadeh "RotationInvariant Multiresolution Texture Analysis Using Radon and Wavelet Transforms" IEEE Transactions on Image Processing, Vol. 14, No. 6, pp783-795, June 2005. 
[19] B. Marcel, M. Briot, and R. Murrieta, "Calcul de translation et rotation par la transformation de Fourier," Traitement du Signal, vol. 14, no. 2, pp. 135-149, 1997.

[20] Salah Althloothi, Mohammad H. Mahoor, Richard M. Voyles" A Robust Method for Rotation Estimation Using Spherical Harmonics Representation" IEEE Transactions on Image Processing, Vol. 22, No. 6, June, 2013.

[21] Shubing Wang, "Application of Fourier transform to imaging analysis" University of Wisconsin-Madison, dept of statistics, 2007.
[22] Sung Cheol Park; Min Kyu Park; Moon Gi Kang; "Super-resolution image reconstruction: a technical overview," Signal Processing Magazine, IEEE , vol. 20, No.3, pp. 21- 36, May 2003

[23] Yunsang Han and Sangkeun Lee, "Parameter Estimationbased Single Image Super Resolution," 1st IEEE Global Conference on Consumer Eletronics (GCCE 2012), pp.565-569, Feb. 2012. 